\title{
Evaluation of Systemic Effects of Negative Pressure Wound Therapy in Open Wound Treatment in Dogs
}

\author{
Rebecca Albert $^{1} \quad$ Sven Reese ${ }^{2}$ Mirja C. Nolff ${ }^{1} \quad$ Andrea Meyer-Lindenberg ${ }^{1}$ \\ ${ }^{1}$ Clinic for Small Animal Surgery and Reproduction, \\ Ludwig-Maximilians University, Munich, Germany \\ 2 Department for Basic Veterinary Sciences, Ludwig-Maximilians \\ University, Munich, Germany \\ Address for correspondence Rebecca Albert, Clinic for Small Animal \\ Surgery and Reproduction, Ludwig-Maximilians-University, \\ Veterinärstrasse 13, 80539 Munich, Germany \\ (e-mail: rebecca.albert@chir.vetmed.uni-muenchen.de).
}

VCOT Open 2019;2:e1-e8.

\begin{abstract}
\section{Keywords}

- negative pressure wound therapy

- polyurethane foam

- C-reactive protein

- prognostic values for wound closure

Objectives The aim of this study was to evaluate the systemic effect of negative pressure wound therapy (NPWT) on the treatment of complicated wounds in dogs. Materials and Methods Dogs undergoing open wound treatment were randomly assigned to one of two groups: NPWT $(n=11)$ or polyurethane foam dressing $(n=11)$. Rectal temperature, heart rate as well as haematocrit, thrombocytes, leucocyte count, band neutrophils, C-reactive protein ( $\mathrm{CrP})$, total protein and albumin were recorded daily from the beginning of therapy (day 0 ) until day 5 , as well as on day 10 . The effect of treatment on systemic parameters was evaluated as well as the prognostic power of the individual parameters with regard to successful wound closure.

Results A more profound systemic decrease was found in total protein under NPWT. This difference was non-significant. Patients with non-successful closure displayed a non-significant trend towards lower initial leukocyte and thrombocyte counts and significantly higher $\mathrm{CrP}$ values on days 4,5 and $10(p<0.05)$ compared with successfully treated patients. Receiver operating characteristic analysis revealed an optimal cutoff value of $70.2 \mathrm{mg} / \mathrm{L}$ at day 4 (sensitivity 80 ; specificity of 85.7 ).

Conclusion There might be an increased loss of protein in NPWT-treated patients, which does not affect albumin levels, otherwise no systemic effects were detected compared with the control treatment. The parameter with the best sensitivity and specificity to detect serious complications (no wound closure achieved) was $\mathrm{CrP}$ at day 4.
\end{abstract}

\section{Introduction}

The first phase of wound healing, namely the inflammatory phase, involves three major components for healing: inflammatory cells, inflammatory mediators and thrombocytes. ${ }^{1,2}$ The process is quite similar between species, ${ }^{2}$ but differences in healing capacity have been described for different species including dogs and cats, as well as horse and ponies. ${ }^{3-6}$ The current knowledge of physiological and delayed wound healing in dogs has mainly been transferred from facts generated in humans, ${ }^{7}$ with a lack of species-specific pathophysiological studies of healing. Studies in humans and pigs have suggested that negative pressure wound therapy (NPWT) exerts profound effects on the wound including cytokine-and even gene modification. ${ }^{8,9}$ The systemic effect of this therapy is still under debate with a very few studies investigating this aspect. A study of diabetic ulcers in man found no effect on systemic leucocytes with a beneficial effect on C-reactive protein $(\mathrm{CrP})$ development under therapy. ${ }^{10}$ Interestingly, clinical trials in dogs and cats suggest that there might be an impact on systemic infection, especially development of systemic signs of systemic inflammatory response syndrome and sepsis in patients treated with NPWT. Nolff and colleagues retrospectively detected improved infection control and fewer cases received

July 30, 2018

accepted after revision

May 2, 2019
DOI https://doi.org/

10.1055/s-0039-1693007. ISSN 2625-2325. (c) 2019 Georg Thieme Verlag KG
Stuttgart · New York

License terms

(1)(1) 
with progressing signs of sepsis under NPWT compared with polyurethane foam treatment in dogs and cats. ${ }^{11,12}$ But so far, there are no prospective randomized studies available to underline and quantify this effect. It is generally accepted that a wound is a local problem that should have little systemic impact, ${ }^{1}$ which might be one of the reasons why systemic impacts of treatment are not frequently investigated. However, previous studies demonstrated that one of the main reasons for open wound treatment in dogs and cats is uncontrollable infection frequently linked with systemic signs of sepsis. ${ }^{11,12}$ In contrast to chronic wounds of other origin, this subtype of wounds represents a group in which systemic effects frequently complicate the situation. Failure to control local and systemic infection are the main reasons for unsuccessful treatment of these patients. ${ }^{11,12}$

Wound infection-especially early stages-is not always easily differentiated from normal healing. The presence of bacteria alone is not helpful, since the bacterial bioburden can be present without major harm of the patient. ${ }^{13}$ Therefore, it seems reasonable to investigate if there are systemic factors, measurable in a clinical examination or in a standard blood test, which might be influenced by treatment-and furthermore-if any of those actually qualify as prognostic factors to identify ongoing infection and potentially predict unsuccessful treatment. This is why we decided to restrict our focus for systemic inflammation on the following parameters. The aim of this study was to evaluate the effect of NPWT on several systemic parameters and to compare it to a foam dressing treatment protocol to identify differences. Furthermore, the prognostic value of rectal temperature, heart rate, leucocyte count, band neutrophil count, haematocrit, thrombocyte count, total protein, albumin and $\mathrm{CrP}$ on day $0-5$ and 10 was evaluated. The hypothesis was that NPWT would positively influence several systemic parameters such as signs of infection. Furthermore, we suggested that prolonged signs of infection such as progressive leucocytosis, $\mathrm{CrP}$ increase, an increase in temperature or heart rate would be related to unsuccessful wound closure after open wound treatment and if one or more of these parameters could be employed as a prognostic factor for a successful wound healing.

\section{Materials and Methods}

The study was performed between July 2014 and September 2016 after approval of the ethic commission of the faculty (22-27-02-2014). Dogs were included if they were presented with injuries requiring open wound treatment due to traumatic tissue loss or infection if previous attempts of closure had failed. Patients were assigned to one of two treatment groups using random numerical lists generated by Excel (Microsoft Corporation; Redmond, Washington, United States) ( - Supplementary Appendix Table 1, available in online version only).

\section{Treatment Protocols}

After owner consent, the patients underwent a general clinical examination, and further diagnostic steps if necessary. A haematology, serum chemistry profile as well as $\mathrm{CrP}$ were obtained from all patients before initiation of therapy. All wounds were then debrided and lavaged, while the patient was under general anaesthesia using polyhexanide biguanide (Prontovet; B. Braun, Melsungen, Germany). In Group A, NPWT was performed at a continuous pressure of $-125 \mathrm{~mm} \mathrm{Hg}$ (V.A.C. GranuFoam; TracPad; ActiVAC, KCI, Wiesbaden, Germany). In Group B, the wounds were initially covered using a foil-coated foam dressing (ALLEYVIN Cavity; Smith \& Nephew GmbH, Hamburg, Germany) sutured to the surrounding skin and changed as needed. Once wound exudation had decreased to a level which allowed the dressings to be left in place for more than 24 hours, the dressing was changed to silver-coated foam (Acticoat Moisture Control; Smith \& Nephew $\mathrm{GmbH}$ ). Planned dressing changes were performed every 3 days during the first 9 days, and every 2 to 3 days thereafter in both groups. All patients received treatment with amoxicillin/clavulanic acid at a dose of $12.5 \mathrm{mg} / \mathrm{kg}$ three times a day (intravenously as long as venous catheters were present, followed by oral administration). Antibiotic treatment was adjusted based on the results of microbiological culture if needed. Analgesia was performed based on the degree of the injury either using fentanyl-ketamine-lidocaine constant rate infusion for 3 days followed by transdermal fentanyl or a pure fentanyl constant rate infusion for 12 hours, followed by transdermal fentanyl. In cases where prolonged analgesia was needed, this was achieved by administering tramadol orally ( $5 \mathrm{mg} / \mathrm{kg}$ three times a day). No non-steroidal drugs or cortisone were permitted in any patient during treatment. Additional treatment was performed as needed based on the severity of the condition of the patients.

\section{Special Investigated Parameters}

The following parameters were assessed at day 0 to 5 as well as day 10: rectal body temperature $\left({ }^{\circ} \mathrm{C}\right)$, heart rate $(\mathrm{BPM})$, leucocyte count (109/L), band neutrophil count (109/L), CrP (mg/L), haematocrit (\%), thrombocyte count (109/L), total protein $(\mathrm{g} / \mathrm{L})$ as well as albumin $(\mathrm{g} / \mathrm{L})$. Complications were defined as severe local deterioration of the wound, increased exudation, increased infection or necrosis of surrounding areas or systemic infections precluding closure under therapy. If successful management of the wounds was not achieved under the randomized treatment option, treatment was changed to the most successful potential alternative $($ foam $\rightarrow$ NPWT, NPWT $\rightarrow$ NPWT with instillation of a solution, NPWT $\rightarrow$ amputation in case of a limb injury) and patients were classified as 'unsuccessful closure achieved under therapy'. Wounds were considered successfully treated if complete closure was achieved and no complications necessitating further surgical treatment developed within 6 weeks after closure.

\section{Statistical Analysis}

Statistical analysis was performed using G-power 3.1 (G-Power 3.1; Heinrich Heine University, Düsseldorf, Germany), BIAS for windows 11 (BIAS for windows 11; Goethe University, Frankfurt, Germany), SPSS (SPSS 20.0; IBM, Munich, Germany) and MedCalc (MedCalc Software; Ostend, Belgium). Effect size (Cohen's-d) and power were calculated for all parameters with regard to treatment (NPWT vs. foam) and wound closure (closure vs. non-closure). Parameters with an 
appropriate power $(>0.8)$ were tested for normality using Shapiro-Wilk test. Significance was set at $p<0.05$ and evaluated using $t$-test for independent means in normally distributed data. Non-normally distributed data were tested using the Mann-Whitney U test. Finally, cutoff values were determined using receiver operating characteristic analysis for parameters that reached significance (-Fig. 1).

\section{Results}

A total of 21 dogs with 22 wounds located at the different body areas including the trunk as well as the extremities were enrolled within the study. One dog developed two separate wounds at different time points and was thus included twice, resulting in a total of 11 wounds included per group. There were no significant differences between groups regarding the mean age (NPWT: 69 months [range: 8-160 months], foam: 92 months [range: 54-152 months]), weight (NPWT: $40.6 \mathrm{~kg}$ [range: $5.5-93 \mathrm{~kg}$ ], foam $40.8 \mathrm{~kg}$ [range: $10.8-93 \mathrm{~kg}$ ]) or wound localization. The majority of patients showed signs of local infection on initial wound evaluation (NPWT 9/11, foam 8/11) and nine patients per group had positive initial bacterial cultures. Further details regarding the patients and wound types can be found in -Supplementary Appendix Table 1 (available in online version only).
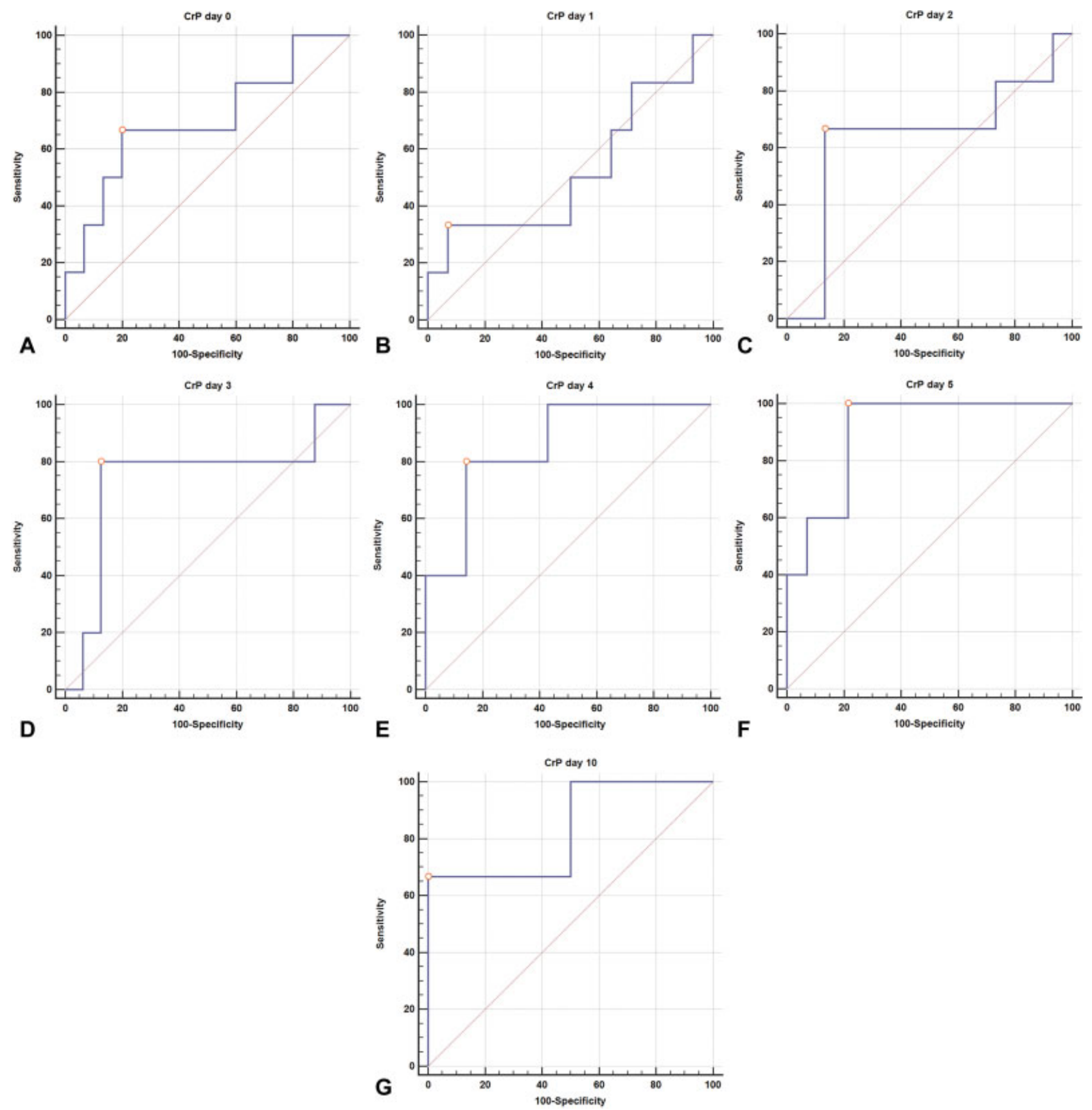

Fig. 1 Receiver operating characteristic analysis illustrating sensitivity and specificity of C-reactive protein (CrP) on day 0 (A), day 1 (B), day 2 (C), day 3 (D), day $4(\mathrm{E})$, day $5(\mathrm{~F})$ and day $10(\mathrm{G})$ with respect to closure. $\mathrm{CrP}$ at day 4 yielded the most reliable combined sensitivity and specificity. 


\section{Comparison of Systemic Parameters within Treatment Groups}

None of the parameters tested showed significant differences within the treatment groups. Patients in both groups initially showed mild leucocytosis and mildly increased band neutrophil counts, especially at days 1 and 2 after surgery, which normalized during the course of treatment in both groups. In contrast to leucocytes, mean $\mathrm{CrP}$ increase was profound, with a peak after the initial debridement and slow decrease during the time of the study; however, in contrast to leucocytes, mean $\mathrm{CrP}$ did not reach normal values during the course of observation. Total protein already showed a minimal decrease in the NPWT group at day 0 , and further decreased mildly in both groups during the observation period. Mean total protein as well as albumin on day 0 was within normal limits with a slow decrease in both groups with a nadir at day 5 followed by an increase at day 10 . Interestingly, total protein was the parameter with the largest detected treatment associated effect (Cohen's-d between 0.6 and 1 ) at all days, while no effect of treatment (Cohen's-d 0-0.2) was detectable regarding albumin development throughout the whole time. Due to the relative small detected effects, none of the tested parameters reached a sufficient power $(>0.8)$ for further testing. However, within the group of tested parameters, the largest effects of therapy were detected for total protein.

\section{Comparison of Systemic Parameters With Regard to Closure}

Wound closure under initial therapy was achieved in 16/22 wounds. Six out of twenty wounds developed serious complications precluding closure under the chosen regimen. Within the wounds treated with NPWT, one dog developed severe thromboembolism of the entire vasculature of the lower limb and paw, resulting in necrosis as confirmed by histopathology. Therapy was discontinued at day 13 and the limb was amputated. The second patient had a severe soft tissue infection and osteomyelitis of the distal phalanx bone of the fourth left hind digit. The dog removed the dressing and caused severe automutilation during therapy; therefore, high amputation of the toe followed at day 32 . The wound finally healed, but the dog developed septic myocarditis and died.

Within wounds treated with a foam dressing, therapy had to be discontinued due to progressive wound infection at day 4 in one patient, as well as due to severe ongoing panniculitis and systemic sepsis in two more patients at days 7 and 10. In the fourth patient, large volumes of wound discharge necessitated multiple dressing changes per day. As wound excretion did not decline by day 10 and no real granulation occurred, this patient was removed from the study and transitioned to NPWT treatment, resulting in decline of discharge and smooth granulation of the wound. One patient in each group died due to wound-related causes (severe infection), while in both groups one patient that was successfully treated died due to unrelated reasons (intraabdominal haemorrhage and end-stage cardiac disease) at 3 and 6 months after wound closure.

The majority of patients showed signs of local infection (17/23) and positive bacterial culture, on initial wound evaluation with no significant differences detected between groups. Mean leucocyte counts were increased at day 0 and 1 in patients in which closure was successful, while they stayed within normal limits in patients where no closure was achieved. Band neutrophils also tended to be higher in patients with successful closure, indicating a more profound immune reaction. However, neither the leucocyte count at day 1 nor the band neutrophil count at days 1 and 2 was significant (power $>0.85$ ). In contrast to this, an initial increase in $\mathrm{CrP}$ was evident in both groups; however, while patients in which no closure was achieved started with a lower $\mathrm{CrP}$ compared with 'closed' patients, values after debridement at day 0 became comparable, with a trend of constant decrease in patients with successful closure, with slower decrease or even increase in non-closed patients (-Fig. 2). The difference in CrP levels in patients with no closure was achieved was significant for day 4 , 5 and $10(p<0.05)$. The highest sensitivity $(80)$ and specificity (85.71) were reached for a cutoff value of $>70.2 \mathrm{mg} / \mathrm{L}$ at day 4 . Cutoff values at day 5 and day 10 did not reach equally reliable predictive values for positive and negative outcome (-Table 1).
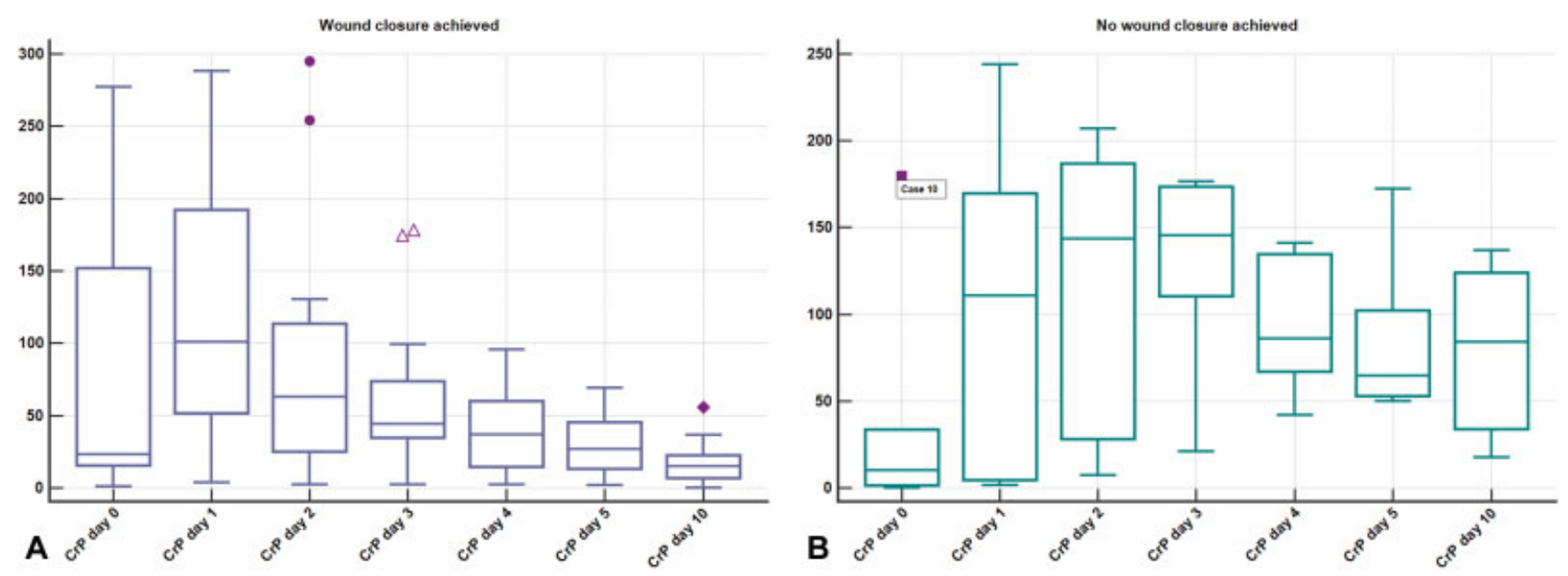

Fig. 2 Boxplot graphs of $\mathrm{C}$-reactive protein ( $\mathrm{CrP})$ development in patients with successful wound closure $(\mathrm{A})$ and those patients where no closure was achieved (B). 


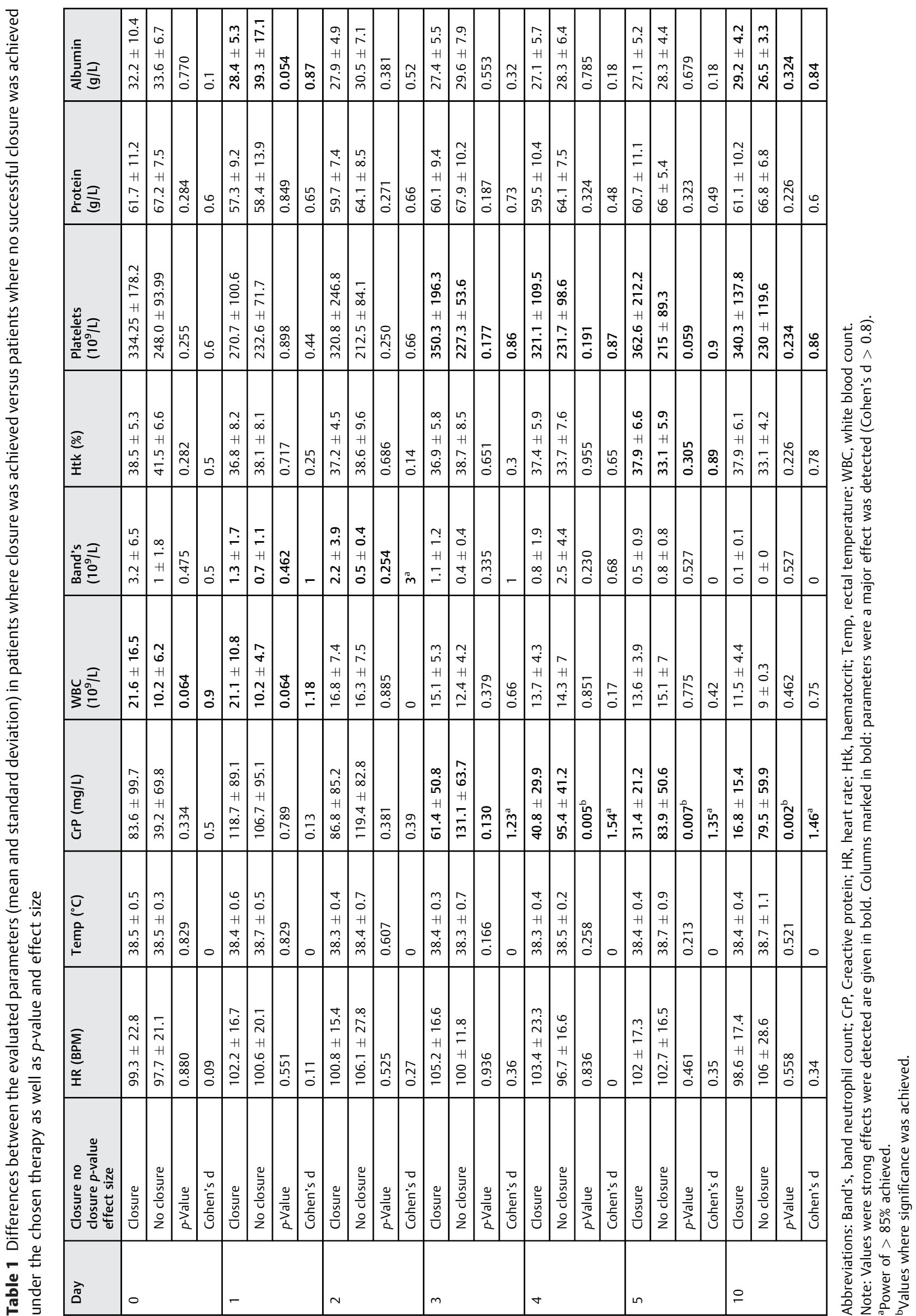


Table 2 Youden index J, associated criterion, sensitivity, specificity and significance level for CrP differed by day

\begin{tabular}{|l|l|l|l|l|l|}
\hline Day & Youden index J & $\begin{array}{l}\text { Associated criterion } \\
\text { (CrP mg/dL) }\end{array}$ & Sensitivity & Specificity & $\begin{array}{l}\text { Significance level of } \\
\boldsymbol{p} \text {-value (area 0.5) }\end{array}$ \\
\hline 0 & 0.4667 & $<15.26$ & 66.67 & 80.00 & 0.1692 \\
\hline 1 & 0.2619 & $<4.4$ & 33.33 & 92.86 & 0.8859 \\
\hline 2 & 0.5333 & $>130.4$ & 66.67 & 86.67 & 0.4216 \\
\hline 3 & 0.6750 & $>99.1$ & 80 & 87.5 & 0.1546 \\
\hline 4 & 0.6571 & $>70.2$ & 80 & 86.71 & 0.0002 \\
\hline 5 & 0.7857 & $>45.1$ & 100 & 78.57 & $>0.0001$ \\
\hline 10 & 0.6667 & $>55.8$ & 66.67 & 100 & 0.054 \\
\hline
\end{tabular}

Abbreviation: CrP, C-reactive protein.

Thrombocytes were lower in non-closed patients at all time points. Protein and albumin both showed mild decreases during treatment; however, values tended to be higher in non-closed patients. A summary of mean values, standard deviation, effect size and $p$-value reached for all tested parameters can be found in - Table 2 .

\section{Discussion}

To consider the fact that open wounds can be very heterogeneous in size, location and presence of infection, the wounds were treated as assigned by randomization. To minimize the diversity of wounds and to keep the groups comparable, we tried to include patients with comparable wounds, according to localization and origin ( - Supplementary Appendix Table 1, available in online version only), so that there was no kind of over- or under-representation of a particular type of wound.

The aim of the present study was to evaluate the systemic effect of NPWT for the treatment of complicated wounds.

Based on the results of the present study, we cannot fully support the hypothesis that NPWT would positively influence signs of systemic infection. This finding is especially interesting compared with previous studies, which documented fewer cases with progressing systemic inflammation under NPWT treatment compared with foam. ${ }^{12}$ This might have been overestimated previously due to study design (retrospective nature, lack of randomization). However, in contrast to the retrospective reports ${ }^{12,14}$ only few patients in our study showed a systemic inflammation at the beginning or during therapy. It might, therefore, also be possible that systemic effects of NPWT might become more obvious in patients that already exhibit more profound systemic signs at initiation of therapy.

We were only able to detect minor effects of NPWT for all tested parameters with exception of protein loss; therefore, this parameter might be worth further investigation. There is currently only one additional study available that investigated protein losses under NPWT treatment in humans. ${ }^{15}$ The authors recorded a median total protein loss of $25 \pm 17 \mathrm{~g} / \mathrm{d}$ in patients treated with NPWT for the management of open abdomens and $8 \pm 5 \mathrm{~g} / \mathrm{d}$ for soft tissue wounds. Unfortunately, these authors did not correlate total protein losses in wound exudates to systemic values. ${ }^{15}$ It is questionable, if the observed systemic difference in total protein in our study is really related to NPWT treatment, or rather to the fact that the mean protein score at day 0 was lower in NPWT-treated patients compared with the foam treated patients. It is, therefore, not certain if the observed lower total protein in NPWT was really related to the therapy regimen or rather represents an effect of uneven randomization between groups.

Our second hypothesis was that prolonged systemic signs of inflammation would be present in patients where successful closure was not supported. Wound healing is a complex process of inflammation followed by proliferation and tissue maturation. ${ }^{1,2}$ While inflammation plays a major part in initiation of this cascade, it becomes detrimental if it persists. $^{16}$ There are three components representing the cornerstones for successful healing: cells of the immune system (e.g. polymorphonuclear leucocytes, monocytes, macrophages, mast cells, T-cells), inflammatory pathways (e.g. interleukin-1 $\beta$, tumour necrosis factor) as well as the coagulation system (e.g. thrombocytes, contact activation pathway, tissue factor pathway). ${ }^{2,16}$ With regard to this knowledge, we found some interesting trends: patients with higher initial leucocyte, band neutrophil counts and thrombocyte counts were more likely to achieve closure.

Leucocytes have an important role in wound healing: they prevent spread of infection by bacterial decontamination and initiate the healing response by secretion of mediators important for fibroblast recruitment. ${ }^{1,2,16,17}$ After activation of the complement system, platelet degranulation and ongoing bacterial degradation attract neutrophils to the scene. ${ }^{18}$ The observed phenomenon of initially higher counts of leucocytes in patients with successful closure might indicate a better or faster immune response leading to a positive effect on subsequent wound healing. Similar observations have been made in horses before, ${ }^{3}$ but not in dogs. In ponies, leucocytes produce more inflammatory mediators, resulting in improved local defence, faster cellular debridement, and a faster transition to the repair phase, with more wound contraction. ${ }^{3}$ Our results may indicate that delayed and insufficient leucocyte recruitment might be one of the steps leading to progressing local infection and delayed healing. However, further studies elucidating the principal pathways and effects on healing in dogs would be needed to verify this finding. 
Besides inflammatory cell types, the coagulation cascade is an integral part of the inflammatory phase. ${ }^{2}$ While these cells are mostly recognized due to their role in haemostasis, they serve as an important source for over 300 different growth factors including chemokines and cytokines (platelet derived growth factor, transforming growth factor- $\beta 1$ and $\beta 2$, fibroblast growth factor, epithelial growth factor etc.). ${ }^{19}$ Activated thrombocytes modulate phagocytosis, production of neutrophil extracellular traps and oxidative burst in neutrophils, thereby contributing to the host immune reaction. ${ }^{20}$ Furthermore, they modulate early events in wound healing including neovascularization and recruitment of fibroblasts. ${ }^{19,21}$ Finally, platelets localize and adhere to sites of bacterial lesions. ${ }^{20}$ Many of their cytokines actually have direct antibacterial effects and activated platelets can directly bind and internalize bacteria themselves. ${ }^{20,22}$ In the present study, thrombocyte counts were decreased in patients who did not achieve closure, and although we did not reach sufficient power to investigate if this was significant, a major effect was detected. Thrombocytopenia indicates recruitment towards the focus of infection and has been documented in severe bacterial infections in humans before. ${ }^{21}$ Our finding that patients with non-successful closure exhibited lower systemic thrombocyte counts might therefore indicate a more severe state of infection combined with a depletion of thrombocytes. However, one major limitation of this study is that we did not correlate systemic and local levels of leucocytes or thrombocytes and their mediators. Further studies seem warranted to further elucidate this interesting connection. While the detected changes in leucocyte and thrombocyte counts were only trends, significant effects were detected for CrP. Contrary to inflammatory cells, the acute phase protein $\mathrm{CrP}$ is a product of inflammation that does not exert any positive effect on wound healing itself, but rather acts as a marker for the severity of inflammation caused by infectious and non-infectious origin. ${ }^{23}$ C-reactive protein is one of the major acute phase proteins in dogs. ${ }^{24-26}$ It is a pattern recognition molecule that binds to pathogens (bacterial-membrane) and cells undergoing death. ${ }^{23}$ It is mainly generated by the liver where the production of positive acute phase proteins is increased, while albumin synthesis is decreased by inteleukin- 6 and interleukin $1 \beta .^{23}$ Besides the liver, small amounts can also be secreted by activated monocytes and lymphocytes. ${ }^{23}$ Aggregated $\mathrm{CrP}$ efficiently activates the complement system through direct interaction with the classic pathway, which is triggered by activation of the C1-complex and enhances phagocytosis, and thus represents an important part of the first line of defence in the host. ${ }^{23}$ One major advantage of $\mathrm{CrP}$ is that it rises markedly and rapidly once infection is present or after surgical trauma. ${ }^{24,27}$ Numerous authors have advocated the inclusion of $\mathrm{CrP}$ in standard inflammatory panels in dogs, as it reacts quicker and more profoundly than leucocytes. ${ }^{25,27,28}$ As seen in previous studies investigating pyometra or sepsis, ${ }^{26,27,29,30}$ we were able to detect that $\mathrm{CrP}$ more reliably documented the failure to control local infection and subsequent non-successful wound closure compared with leucocytes. We also found that it is not only the absolute value at the beginning of therapy, but rather the development over time that made the difference. Similar to Dabrowski and colleagues, who investigated $\mathrm{CrP}$ as a marker for surgical site infections after pyometra surgery in bitches ${ }^{31}$, we found that values estimated at day 4 had the highest prognostic value. This is in part due to the fact that $\mathrm{CrP}$ is increased by surgical trauma, it will rise as fast as 6 hours after surgery and peak around 12 to 24 hours after completion. ${ }^{31,32}$ Therefore, monitoring $\mathrm{CrP}$ at day 1 is of little value if taken alone; however, if taken as a reference value for further development, it might become useful. Based on the results of our study, laboratory evaluations at day 1 and 4 after surgery seem most warranted for monitoring of inflammation.

It is interesting that total protein and albumin values did not differ markedly between patients with successful or non-successful closure, even if they represent one of the factors known to influence wound healing. ${ }^{2}$ This might be due to the fact that these values will only be altered in severely ill patients, and are thus not sensitive enough to mirror subtle changes in local wound infections. There are several limitations to this study, including sample size, differences in wounds (localization, reason, size) and the lack to correlate systemic and local factors, which should be addressed in future studies. Investigation of wound exudate in dogs might be warranted, as it might resemble a more accurate and less invasive source for monitoring of local processes.

\section{Conclusion}

From the results of this study, we conclude that several parameters seem interesting regarding further investigation of their prognostic power in wound closure, including leucocyte and thrombocyte count as well as total protein. Among the tested parameters, $\mathrm{CrP}$ seems to be the most valuable parameter regarding the prediction of successful wound closure, with a cutoff value of $70.2 \mathrm{mg} / \mathrm{L}$ at day 4 reaching the highest sensitivity and specificity.

\section{Author Contribution}

Rebecca Albert and Mirja C. Nolff contributed to conception of study, study design, acquisition of data and data analysis and interpretation. Sven Reese contributed to data analysis and interpretation. Andrea Meyer-Lindenberg contributed to conception of study and study design. Rebecca Albert and Mirja C. Nolff and Andrea MeyerLindenberg drafted, revised and approved the submitted manuscript.

\section{Conflict of Interest}

The senior author acts as key opinion leader for Kinetic Concepts Inc. Germany. Kinetic Concepts Inc. was not involved in any part of this study; no financial associations exist.

\section{Acknowledgment}

The authors want to thank the 'Gesellschaft zur Förderung Kynologischer Studien' for partly funding the patients involved in this study. 


\section{References}

1 Pavletic MM. Wound management principles and techniques. Vet Q 1997;19(Suppl 1):22-24

2 Gurtner GC, Werner S, Barrandon Y, Longaker MT. Wound repair and regeneration. Nature 2008;453(7193):314-321

3 Wilmink JM, van Weeren PR. Second-intention repair in the horse and pony and management of exuberant granulation tissue. Vet Clin North Am Equine Pract 2005;21(01):15-32

4 Wilmink JM, Nederbragt H, van Weeren PR, Stolk PW, Barneveld A. Differences in wound contraction between horses and ponies: the in vitro contraction capacity of fibroblasts. Equine Vet J 2001; 33(05):499-505

5 Bohling MW, Henderson RA, Swaim SF, Kincaid SA, Wright JC. Comparison of the role of the subcutaneous tissues in cutaneous wound healing in the dog and cat. Vet Surg 2006;35(01):3-14

6 Bohling MW, Henderson RA, Swaim SF, Kincaid SA, Wright JC. Cutaneous wound healing in the cat: a macroscopic description and comparison with cutaneous wound healing in the dog. Vet Surg 2004;33(06):579-587

7 Volk SW, Bohling MW. Comparative wound healing-are the small animal veterinarian's clinical patients an improved translational model for human wound healing research? Wound Repair Regen 2013;21(03):372-381

8 Glass GE, Murphy GF, Esmaeili A, Lai LM, Nanchahal J. Systematic review of molecular mechanism of action of negative-pressure wound therapy. Br J Surg 2014;101(13):1627-1636

9 Nuutila K, Siltanen A, Peura M, et al. Gene expression profiling of negative-pressure-treated skin graft donor site wounds. Burns 2013;39(04):687-693

10 Dzieciuchowicz Ł, Kruszyna Ł, Krasiński Z, Espinosa G. Monitoring of systemic inflammatory response in diabetic patients with deep foot infection treated with negative pressure wound therapy. Foot Ankle Int 2012;33(10):832

11 Nolff MC, Fehr M, Reese S, Meyer-Lindenberg AE. Retrospective comparison of negative pressure wound therapy and silvercoated foam dressings in open-wound treatment in cats. J Feline Med Surg 2017;19(06):624-630

12 Nolff MC, Fehr M, Bolling A, et al. Negative pressure wound therapy, silver coated foam dressing and conventional bandages in open wound treatment in dogs. A retrospective comparison of 50 paired cases. Vet Comp Orthop Traumatol 2015;28(01):30-38

13 Weed T, Ratliff C, Drake DB. Quantifying bacterial bioburden during negative pressure wound therapy: does the wound VAC enhance bacterial clearance? Ann Plast Surg 2004;52(03):276-279 , discussion $279-280$

14 Stanley BJ, Pitt KA, Weder CD, Fritz MC, Hauptman JG, Steficek BA. Effects of negative pressure wound therapy on healing of free fullthickness skin grafts in dogs. Vet Surg 2013;42(05):511-522

15 Hourigan LA, Hourigan L, Linfoot JA, et al. Loss of protein, immunoglobulins, and electrolytes in exudates from negative pressure wound therapy. Nutr Clin Pract 2010;25(05):510-516

16 Eming SA, Krieg T, Davidson JM. Inflammation in wound repair: molecular and cellular mechanisms. J Invest Dermatol 2007;127 (03):514-525
17 Pitzer GB, Patel KG. Proper care of early wounds to optimize healing and prevent complications. Facial Plast Surg Clin North Am 2011;19(03):491-504

18 Grose R, Werner S. Wound-healing studies in transgenic and knockout mice. Mol Biotechnol 2004;28(02):147-166

19 Golebiewska EM, Poole AW. Platelet secretion: from haemostasis to wound healing and beyond. Blood Rev 2015;29(03): 153-162

20 Jenne CN, Urrutia R, Kubes P. Platelets: bridging hemostasis, inflammation, and immunity. Int J Lab Hematol 2013;35(03): 254-261

21 McNicol A, Israels SJ. Beyond hemostasis: the role of platelets in inflammation, malignancy and infection. Cardiovasc Hematol Disord Drug Targets 2008;8(02):99-117

22 Yeaman MR. Platelets: at the nexus of antimicrobial defence. Nat Rev Microbiol 2014;12(06):426-437

23 Black S, Kushner I, Samols D. C-reactive protein. J Biol Chem 2004; 279(47):48487-48490

24 Kjelgaard-Hansen M, Strom H, Mikkelsen LF, Eriksen T, Jensen $\mathrm{AL}$, Luntang-Jensen $\mathrm{M}$. Canine serum C-reactive protein as a quantitative marker of the inflammatory stimulus of aseptic elective soft tissue surgery. Vet Clin Pathol 2013;42(03): 342-345

25 Kjelgaard-Hansen M, Jacobsen S. Assay validation and diagnostic applications of major acute-phase protein testing in companion animals. Clin Lab Med 2011;31(01):51-70

26 Christensen MB, Eriksen T, Kjelgaard-Hansen M. C-reactive protein: quantitative marker of surgical trauma and post-surgical complications in dogs: a systematic review. Acta Vet Scand 2015; 57:71

27 Christensen MB, Langhorn R, Goddard A, et al. Comparison of serum amyloid $A$ and $C$-reactive protein as diagnostic markers of systemic inflammation in dogs. The Canadian veterinary journal. Can Vet J 2014;55(02):161-168

28 Cerón JJ, Martinez-Subiela S, Ohno K, Caldin M. A seven-point plan for acute phase protein interpretation in companion animals. Vet J 2008;177(01):6-7

29 Karlsson I, Wernersson S, Ambrosen A, et al. Increased concentrations of C-reactive protein but not high-mobility group box 1 in dogs with naturally occurring sepsis. Vet Immunol Immunopathol 2013;156(1-2):64-72

30 Gebhardt C, Hirschberger J, Rau S, et al. Use of C-reactive protein to predict outcome in dogs with systemic inflammatory response syndrome or sepsis. J Vet Emerg Crit Care (San Antonio) 2009;19 (05):450-458

31 Mathon DH, Palierne S, Meynaud-Collard P, et al. Laparoscopicassisted colopexy and sterilization in male dogs: short-term results and physiologic consequences. Vet Surg 2011;40(04): 500-508

32 Nevill B, Leisewitz A, Goddard A, Thompson P. An evaluation of changes over time in serum creatine kinase activity and C-reactive protein concentration in dogs undergoing hemilaminectomy or ovariohysterectomy. J S Afr Vet Assoc 2010;81(01): $22-26$ 